\title{
SHORT
COMMUNICATIONS
}

\section{Improved Synthesis of Optically Active Ipsdienol}

\author{
V. N. Kovalenko ${ }^{a}$ and K. N. Prokhorevich ${ }^{b}$ \\ ${ }^{a}$ Belarusian State University, pr. Nezavisimosti 4, Minsk, 220030 Belarus \\ e-mail:kovalenkovn@rambler.ru \\ ${ }^{b}$ Belarusian State Medical University, Minsk, Belarus
}

Received October 19, 2015

\section{DOI: $10.1134 / \mathrm{S} 1070428016050250$}

In our preceding publication [1] a simple procedure was described for the preparation of both enantiomers of monoterpene alcohol ipsdienol 1, an actual pheromone component of bark beetle of genus Ips. The key synthesis stage was the resolution of the direct precursor of the ipsdienol, ( \pm )-6-methyl-2-(2chloroethyl)hepta-1,5-dien-4-ol by its conversion into acid phthalate and the crystallization of the acid phthalate as salt with $(+)-$ or $(-)-1$-phenylethylamine. Then the optically active acid phthalates $(S)-2$ and $(R)$ 2 were treated with potassium tert-butylate in tertbutyl alcohol. By the dehydrochlorination and the simultaneous alcoholysis of the ester moiety in one stage $(S)$ - or $(R)$-ipsdienol was obtained in a $55 \%$ yield.

The necessity of preparation of a large amount of optically active ipsdienol $(S)$-1 required a detailed study of the reaction between acid phthalate $(S)$-2 and potassium tert-butylate, since the reaction proceeded with a reproducible yield about $50 \%$. In the initial procedure [1] a large amount of solvent and an essential excess of the base were required. In the reaction mixture a large quantity of unreacted acid phthalate of ipsdienol was present. The increase in the reaction dura-tion from 1 to $3 \mathrm{~h}$ and the use of more concentrated $20 \%$ solution of potassium tert-butylate not only did not increase the yield of ipsdienol $(S)-\mathbf{1}$, but even resulted in a consi-derable decrease in its enantiomeric purity. In various expe-riments we observed unexpected and undesirable decrease in the enantiomeric excess of ipsdienol (S)-1 to 75-80\% (Scheme 1).

The heterolysis of esters of the optically active allylic alcohols at the $\mathrm{O}-\mathrm{R}$ bond ( where $\mathrm{R}$ is the residue of the allylic alcohol) and the accompanying rearrangements with the migration of the double bond and the racemization along the $S_{N} 1$ mechanism are studied in sufficient detail and have been discussed before [2-5]. These transformations were mostly studied by an example of acid phthalates which were used for resolution of the corresponding allylic alcohols into individual enantiomers. It was established by this research that the heterolysis of esters with the formation of allylic carbocations is sharply accelerated at higher temperature, but the optically active allylic alcohols proper do not suffer

\section{Scheme 1.}<smiles>C=C(CCCl)C[C@H](C=C(C)C)OC(=O)c1ccccc1C(=O)O</smiles>

(S)-2

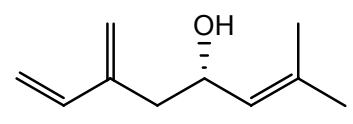

$(S)-1$

1. $t$-BuOK-t-BuOH ( $16.5 \%$ solution), reflux $5 \mathrm{~min}$

2. $\mathrm{MeOH}, 20^{\circ} \mathrm{C}, 60 \mathrm{~h}$

$>70 \%$, ee $>98 \%$ 
racemization and rearrangement in a strongly alkaline environment even at prolonged heating. At $100^{\circ} \mathrm{C}$ the racemization of an ester of an optically active allylic alcohol, trans-pent-3-en-2-yl 4-nitrobenzoate, occurred significantly faster than the hydrolysis of the initial ester [5]. The racemization rate fit to the kinetic law of the first order thus confirming the intramolecular mechanism of the formation of the ion-pair intermediate: The ions constituting the latter recombined giving the racemic 4-nitrobenzoate. In the medium of a protic solvent of a high dielectric permittivity the formation of 4-nitrobenzoate anion and allylic carbocation is expectable. The racemization mechanism was proved using an ester labelled at the carbonyl group with $\mathrm{O}^{18}$ [5]. We may conclude that the phthalate of ipsdienol (S)-1 not yet subjected to alcoholysis may similarly suffer racemization in alcohol with a considerable rate, especially at a high temperature.

The dehydrohalogenation under the action of potassium tert-butylate occurred in a boiling solution practically instantly after mixing the reagents, whereas the alcoholysis of the phthalate group proceeded relatively slowly, and the reaction decelerated even more in concentrated solutions. We presumed that the alcoholysis of the ester may be accelerated by replacement of the sterically hindered tert-butylate by a methylate anion, and the reaction should be carried out at a deliberately low temperature preventing the racemization of the phthalate of ipsdienol $(S)$-1 along the ion-pair mechanism. After the end of the dehydro-chlorination stage which was performed using slightly less concentrated $16.5 \%$ solution of potassium tert-butylate the reaction mixture was at once treated with methanol. The methanolysis of ipsdienol (S)-1 phthalate proceeded without notable difficulties and completed after maintaining the reaction mixture at room temperature for $60 \mathrm{~h}$ in a yield exceeding $70 \%$. The analysis of the ${ }^{1} \mathrm{H}$ NMR spectrum of the Mosher ester of the obtained ipsdienol $(S)$-1 confirmed the complete retention of the optical purity.

Thus we have developed and substantiated the improved version of the formerly advanced transformation providing a possibility to increase the yield of ipsdienol $(S)-\mathbf{1}$ and to prevent the racemization that considerably reduces the preparative value of the reslution of allylic alcohol by crystallization.

(4S)-2-Methyl-6-methylideneocta-2,7-dien-4-ol [(S)-ipsdienol] (S)-1. In $490 \mathrm{~g}$ of anhydrous tert-butyl alcohol at vigorous stirring and boiling was dissolved $29.9 \mathrm{~g}(0.767 \mathrm{~mol})$ of metal potassium. To the obtained solution of potassium tert-butylate heated at $75^{\circ} \mathrm{C}$ at vigorous stirring was added within 10 min a solution of $54.0 \mathrm{~g}(0.160 \mathrm{~mol})$ of acid phthalate $(S)-2$ [1] in $50 \mathrm{~g}$ of anhydrous tert-butyl alcohol. After adding all the substance the mixture was boiled for $5 \mathrm{~min}$ at stirring and afterwards the reaction mixture was quickly cooled to the room temperature $\left(20^{\circ} \mathrm{C}\right)$. The flask was cooled by an ice water bath, and at stirring into the reaction mixture $500 \mathrm{~mL}$ of anhydrous methanol was added. The reaction mixture was left standing at room temperature for $60 \mathrm{~h}$. The reaction mixture was evaporated on a rotary evaporator at a reduced pressure, the residue was diluted with $800 \mathrm{~mL}$ of water, the reaction product was extracted with tertbutyl methyl ether $(4 \times 150 \mathrm{~mL})$. The ether extract was washed with saturated solutions of $\mathrm{NaHCO}_{3}$ and $\mathrm{NaCl}$, dried with calcined $\mathrm{Na}_{2} \mathrm{SO}_{4}$, and evaporated on a rotary evaporator at a reduced pressure. The residue was purified by chromatography on a column charged with $300 \mathrm{~g}$ of silica gel (70-230 mesh), eluting first with petroleum ether (bp $40-60^{\circ} \mathrm{C}$ ), then with its mixtures with ethyl acetate, from $50: 1$ to $10: 1$. Yield $17.1 \mathrm{~g}(70 \%)$. Spectral characteristics and the angle of optical rotation of compounds obtained coincided with the previously measured data [1].

${ }^{1} \mathrm{H}$ and ${ }^{13} \mathrm{C}$ NMR of compounds solutions in $\mathrm{CDCl}_{3}$ were registered on a spectrometer Bruker Avance 400 at operating frequencies 400 and $100 \mathrm{MHz}$ respectively. IR spectra were recorded on a spectrophotometer Vertex 70. Optical rotation was measured on a polarimeter CM-3 at $20 \pm 2^{\circ} \mathrm{C}$. The solvents were dried by standard methods and distilled.

\section{ACKNOWLEDGMENTS}

The study was carried out under a financial support of Forestry Ministry of Belarus' Republic. The authors express their gratitude to Professor R.M. Kellogg ("Syncom B.V.," The Netherlands) for the participation in the discussion of obtained results.

\section{REFERENCES}

1. Kovalenko, V.N. and Matyushenkov, E.A., Russ. J. Org. Chem., 2012, vol. 48, p. 1168.

2. Kenyon, J., Partridge, S.M., and Philips, H., J. Chem. Soc., 1937, p. 207.

3. Duveen, D.I. and Kenyon, J., J. Chem. Soc., 1939, p. 1697.

4. Balfe, M.P. and Kenyon, J., Trans. Faraday Soc., 1941, vol. 37, p. 721.

5. Harlan, L.G. and Melvin, M.P., J. Am. Chem. Soc., 1960, vol. 82, p. 2515. 